\title{
Isomorphic queues
}

\section{A. Ghosal}

Dedicated to P.A.P. Moran for his sixtieth birthday

\begin{abstract}
Two systems of queues are said to be isomorphic in the restricted sense if at least one of their output elements, for example waiting time, queue size, idle time, inter-departure time, and so on, has exactly or approximately the same probability distribution function, irrespective of differences in their input elements, for example, inter-arrival time, service time, and so on. This property has been used in deriving approximate solutions to systems which do not have exact solutions. The paper highlights the systems approach in queueing problems and its practical usefulness.
\end{abstract}

\section{Introduction}

The dictionary meaning of an isomorph is 'equivalent structure'; hence a system $A_{1}$ is an isomorph of another system $A_{2}$ if both have equivalent structures or input-output mechanisms. In the literature of algebra or cybernetics, isomorphy usually implies strictly a one-to-one mapping; in other words, there is a one-to-one correspondence in the input structures as also in the output structures and the input-output relation structures between $A_{1}$ and $A_{2}$. There is no deviation from the dictionary meaning of isomorph if we use it in a restrictive sense: $A_{1}$ is

Received 20 May 1977. The work was done while the author was a Visiting Professor at the Graduate Center of Management at Baruch College, City University of New York. The author is grateful to Professor N. Paul Loomba, Chairman of the Center, for providing facilities for working. He also thanks Professor David G. Kendall, FRS, for encouragement. 
an isomorph of $A_{2}$ with respect to only one or two specific output properties. Talking about two queueing systems $A_{1}$ and $A_{2}$, we may say that $A_{1}$ is a restricted isomorph (R-isomorph) of $A_{2}$ with respect to the distribution function of the waiting time only or with respect to the distribution function of the idle time only. This implies that the two queueing systems (say both are single-server systems) may have different inter-arrival and service time distribution functions but they are equivalent only with respect to the distribution function of the waiting time or idle time. We can call two such systems iso-functional also.

Isomorphic queueing systems (in the restricted sense), which we may call iso-functional as well, have been discussed in this paper. Motivation of this study has been to help in the numerical approximation of the properties of complex queueing systems which do not generally have analytical solutions. The concept of isomorphic queues was introduced by the present author in his studies on the cybernetic modelling of queueing and storage systems (Ghosal $[3,4,5,6]$ ). A more detailed theoretical treatment of the topic along with a few applications are reported in this paper.

\section{Definitions}

A cybernetic system $A$ is defined by a set of elements $X=\left(x_{1}, \ldots, x_{n}\right)$ and a set of mutual relations $R$ among them (KIir and Valach [9], Ghosal $[3,6])$ :

$$
A=\{X, R\} \text {. }
$$

The output of the system $y=\left(y_{1}, \ldots, y_{m}\right)$ is given by the following mapping relationship

$$
f:\{X, R\} \rightarrow Y,
$$

meaning that

$$
f(X ; R)=\cdot Y \text {. }
$$

A system is completely determined if we know the sets $X, R$, and the mapping relation $f$.

When we express a single server queue as a system $A$, we have the 
input

$$
X=(S, T)
$$

where

$S$ represents the service mechanism, implying the service time distribution function, state-dependence, and so on;

$T$ represents the inter-arrival mechanism, implying the distribution function of the inter-arrival time, its statedependence, if any, and so on;

$R$ represents queue discipline (for example, first come first, served, last come first served, priority feature, and so on).

The output is given by

$$
Y=(W, Q, I, B, D)
$$

where

$W$ is the distribution function of the waiting time of a customer at a stationary state or transient state;

$Q$ is the distribution function of the queue size at a particular time, departure epoch, and so on;

$I$ is the idle time distribution function of the server;

$B$ is the distribution function of the length of the successive busy periods;

$D$ is the distribution function of the inter-departure time of customers.

We have the following definitions of isofunctional systems.

DEFINITION 2.1 (Strict Isomorphism). Let the system $A_{i}(i=1,2)$ be defined by

$$
A_{i}=\left\{X_{i}, Y_{i} ; f_{i}\right\}
$$

where $X_{i}$ and $y_{i}$, for the $i$ th system are defined in (2.4) and (2.5), and $f_{i}$ is the mapping relation. The systems are strictly isomorphic if $y_{1}$ and $y_{2}$ are exactly equivalent in distribution functions, so are $X_{1}$ 
and $X_{2}$. This is denoted by

$$
y_{1} \sim y_{2} \text { and } x_{1} \sim X_{2}
$$

where " $\sim$ " stands for equivalence in distribution functions.

REMARKS. The equivalence in distribution functions between $Y_{1}$ and $y_{2}$ implies that the waiting times $W_{1}$ and $W_{2}$ are equivalent in distribution functions, so are queue sizes $Q_{1}$ and $Q_{2}$, and so on - in other words, all output elements in one system are equivalent in distribution functions to the corresponding output elements in the other system.

DEFINITION 2.2 (Restricted Isomorphism). Restricted isomorphism (or $R$-isomorphism) between $A_{1}$ and $A_{2}$ implies that they are equivalent in distribution functions with respect to one or more but not all of the output elements.

REMARKS. The systems $A_{1}$ and $A_{2}$ may have the same waiting time distribution function but may have different idle time distribution functions.

DEFINITION 2.3 (Quasi-isomorphism). Two systems are quasiisomorphic with each other if systems $A_{1}$ and $A_{2}$ have either

(i) approximately the same distribution function for each of the output elements, or

(ii) approximately the same distribution function for one or two of output elements (which is in fact quasi $R$-isomorphism).

The definition of approximately the same distribution function of an output element implies either of the following:

(a) given $F_{i}(x)$ the distribution function of the specific output element, $i=1,2$, we fail to reject the hypothesis $H\left(F_{1}=F_{2}\right) ;$ or

(b) given a small number $\varepsilon(0)$,

$$
\left|F_{1}(x)-F_{2}(x)\right|<\varepsilon, x \in(0, \infty) \text {. }
$$


In many practical problems (2.8) may be a good substitute for (a), particularly when Kolmogoroff-Smirnoff's test is too sensitive.

DEFINITION 2.4 (Isomorph). The system $A_{1}$ is an isomorph of $A_{2}$ or vice-versa if $A_{1}$ and $A_{2}$ are isomorphic in the strict, restricted, quasi- or quasi-restricted sense as per Definitions 2.1, 2.2, and 2.3.

In most of the problems involving approximations of complex queueing processes we shall be concerned with restricted, quasi-, and quasirestricted isomorphs.

\section{Isomorphs of a few one-server models}

In this section we determine isomorphs (in the $R$ - or quasi-R sense) of a few one server models. We shall represent the isomorph in the form of an equivalent $M / M / 1, M / G / 1$, or $M / H / 1$ system ( $H$ implies a hyperexponential distribution). We give the following interpretations by drawing on the results in queueing theory.

THEOREM 3.1. For every $G I / M / 1$ system there exists an $M / M / 1$ $R$-isomorph (with respect to the wating time distribution function).

Proof. Let $A_{I}$ be a $G I / M / I$ system in which the service time $\left(s_{n}\right)$ of the $n$th customer follows the exponential distribution function

$$
\mu \exp (-\mu x) d x \quad(\mu>0 ; x \in(0, \infty)),
$$

and the inter-arrival time follows a general distribution function $B(x)=\operatorname{prob}\left(T_{n} \leq x\right)$, where $T_{n}$ is the interval between the arrival times of the $n$th and $(n+1)$ th customers. Then, if the traffic density $(\rho)$ is less than 1 , the waiting time distribution function $F(x)=\operatorname{prob}(W \leq x)$ is given by

(3.2) $\quad F(0)=1-\delta$ and $d F(x)=(1-\delta) \delta \mu \exp \{-\mu x(1-\delta)\} d x, x \in(0, \infty)$, where $\delta$ is the unique positive root of the equation

$$
K(z)=z,
$$

in which $K(z)=\sum_{r=0}^{\infty} z^{r} k_{r}$, where $k_{r}$ is the probability that $r$ services are completed during a period $(0, x)$, where the origin is arbitrary. The 
result (3.2) is due to $5 \mathrm{mith}$ [13], who proved that the waiting time in a $G I / M / 1$ system follows an exponential distribution. The interpretation of Theorem 3.1 is slight different: from (3.2) it follows that there is an $M / M / 1$ system $\left(A_{2}\right)$ in which the inter-arrival time follows an exponential distribution function with parameter $\delta$ and the service time is the same as that of the system $A_{1}$ (which is a $G I / M / I$ system) - the waiting time distribution function and the queue size distribution function of the two systems are exactly the same.

The inter-departure characteristics of the systems $A_{1}$ and $A_{2}$ are different, because in $A_{2}$ the inter-departure times $(D)$ are independent identically distributed random variables (Finch [1]), while in $A_{1}$ they are not so.

EXAMPLE 3.1.1. There is an $M / M / 1$ isomorph (with respect to the distribution functions of waiting time and queue size) of an $E_{p} / M / 1$ system. If the inter-arrival time follows the distribution function

$$
\left[\alpha^{p} / \Gamma(p)\right] x^{p-1} \exp (-\alpha x) d x \quad(p>0 ; x \in(0, \infty)),
$$

then (3.3) becomes

$$
(1-a)^{p} /(1-a z)^{p}=z
$$

where $a=\mu /(\alpha+\mu)$, the service time following the exponential distribution function with parameter $H$. "The equivalent $M / M / I$ system has the inter-arrival distribution function

$$
\alpha^{\prime} \exp \left(-\alpha^{\prime} x\right) d x, \quad x \in(0, \infty),
$$

where

$$
\alpha^{\prime}=\mu \delta,
$$

where $\delta$ is the positive root of $(3.5)$ on the range $(0,1)$.

THEOREM 3.2. For every $M / G / 1$ system, there exists an $M / M / 1$ R-isomorph with respect to idle time distribution functions.

Proof. Let the $M / G / 1$ system $\left(A_{1}\right)$ have an inter-arrival distribution function $\alpha \exp (-\alpha x) d x \quad(\alpha>0, x \in(0, \infty))$, and the service time follow a general distribution function $G(x)$. Then the idle time follows, under 
equilibrium conditions, the distribution function

$$
\operatorname{prob}(I>x)=C \exp (-\alpha x)
$$

where $C=1-\alpha E(S)$, in which $E(S)$ is the expectation of the service time (Ghosal [3], p. 18, equation 1.49).

Let $A_{2}$ be an $M / M / 1$ system in which the inter-arrival time follows an exponential distribution function with parameter $\alpha$ and the service time follows an exponential distribution function with parameter $\mu$. Then

$$
E(S)=1 / \mu ;
$$

the idle time distribution function of $A_{1}$ is the same as that of $A_{2}$.

THEOREM 3.3. The isomorph (with respect to idle time distribution function) of an $M / G / I$ system does not have equivalent waiting time, queue size, and inter-departure time distribution functions.

Proof. Let $D_{n}$ be the interval between departure times of the $n$th and $(n+1)$ th customers, $I_{n}$ the idle time of the server after serving the $n$th customer; then we have

$$
D_{n}=S_{n}+I_{n}
$$

Since the distribution function of the service time in system $A_{1}(M / G / 1)$ is not the same as that in the system $A_{2}$ (the $M / M / 1 \quad R$-isomorph with respect to idle time distribution function), the distribution function of $D_{n}$ differs in the two systems.

The waiting time distribution function in the system $A_{2}$ follows the exponential distribution function (3.2), in which $\delta=\alpha / \mu$. The waiting time distribution function of the system $A_{1}$ is not exponential; its Laplace transform $\varphi(\theta)$ is given by

$$
\varphi(\theta)=[(1-\delta) \theta] /[\theta-\alpha+\alpha \Phi(\theta)] .
$$

where $\Phi(\theta)$ is the Laplace transform of the service time. While the traffic density $\alpha$ is the same for both the systems $A_{1}$ and $A_{2}, \Phi(\theta)$ are different for two systems. In fact, if $\varphi_{i}(\theta)$ is the Laplace transform of the waiting time distribution function for the $i$ th system, 
$\Phi_{i}(\theta)$ the Laplace transform of the service time distribution function for the $i$ th system $(i=1,2)$, then shifts in mean and variance of two waiting time distributions can be estimated.

\section{Quasi-isomorphs}

In this section we give examples of a few useful quasi-isomorphs for approximation of queueing systems.

\section{$4.1 \quad N / M / 1 \quad$ SYSTEM}

In a few experimental studies done by the present author the interarrival time of a one-server queueing system was found to follow approximately a truncated normal distribution on the range $(0, \infty)$, and the service distribution approximated an exponential distribution. This we call an $N / M / 1$ system, where $N$ stands for the truncated normal distribution (we may also write $T N$ for $N$ ). In one case the mean $\lambda$ was $2.3 \lambda(m)$ times the standard deviation $(\sigma)$. Theorem 3.1 states that the waiting time for such a system follows an exponential distribution (3.2) in which $\delta$ is determined by

$$
z=K(z)=n(\mu-\mu z)
$$

where $n(\cdot)$ is the Laplace transformation of the truncated normal distribution.

For $m>25$, we can assume the Laplace transformation of the whole distribution to be approximately the same as that of the truncated distribution, that is,

$$
\begin{aligned}
n(\mu) & =(c / \sqrt{2 \pi \sigma}) \int_{0}^{\infty} \exp \left[-\mu x+\frac{(x-m)^{2}}{2 \sigma^{2}}\right] d x \\
& =(1 / \sqrt{2 \pi \sigma}) \int_{-\infty}^{\infty} \exp \left[-\mu x+\frac{(x-m)^{2}}{2 \sigma^{2}}\right] d x,
\end{aligned}
$$

where $c$ is a constant for the truncated distribution; hence

$$
n(\mu)=\exp \left[2 m \mu+\sigma^{2} \mu^{2}\right] .
$$

Thus (4.1) leads to

$$
\text { (4.2) } z=\exp \left[2 m \mu(1-z)+\sigma^{2} \mu^{2}(1-z)^{2}\right] \text {. }
$$


The solution of (4.2) by numerical methods gives the root $\delta$ in (3.2). Consequently the $M / M / 1$ equivalent system is a quasi-isomorph.

A few other experimental studies on $N / M / I$ systems have been done by the author and his associates; it is indicated that sometimes, even if $m>1.50$, the error obtained through a quasi-isomorph is not high. The distribution function of the waiting time obtained through the quasiisomorph is tested against the simulated waiting time distribution. Values of $\delta$ for a few $N / M / 1$ distributions have been calculated in Table 4.1 below.

TABLE 4.1

Values of $\delta, E(W)$, and $\operatorname{var}(W)$

\begin{tabular}{l|c|c|c|c|c|c|c}
\hline & & \multicolumn{3}{|c|}{$\mu=0.2$} & \multicolumn{3}{c}{$\mu=0.6$} \\
\cline { 3 - 8 } & & $\delta$ & $E(W)$ & $\operatorname{var}(W)$ & $\delta$ & $E(W)$ & $\operatorname{var}(W)$ \\
\hline 1 & $m=6, \sigma=2$ & 0.715 & 12.53 & 282.3 & 0.065 & 0.115 & 0.398 \\
3 & $m=8, \sigma=2$ & 0.386 & 3.14 & 41.2 & 0.018 & 0.030 & 0.102 \\
& $m=10, \sigma=5$ & 0.326 & 2.42 & 30.0 & 0.156 & 0.308 & 1.121 \\
\hline
\end{tabular}

Calculations are due to Stern [14].

\subsection{QUASI-ISOMORPHS OF $M / G / 1$ SYSTEM}

From (3.8) we find that an $M / M / 1$ isomorph, with respect to the idle time distribution function of the server, of an $M / G / 1$ system will differ in respect of waiting time distribution. It has been proved that the waiting time of an $M / E_{p} / 1$ system follows a generalized hyperexponential distribution

$$
\operatorname{prob}(W>x)=\sum_{i=1}^{k} c_{i} \exp \left(-d_{i} x\right),
$$

where $c_{i}, d_{i}$ may be complex-valued $(i=1, \ldots, k)$.

Proof. Let $\varphi(\cdot)$ be the Laplace transformation of the waiting time distribution function and $\Phi(\cdot)$ that of the service time distribution. Then the traffic density is $\delta=\alpha E(S)$ and $\varphi(\cdot)$ is given by (3.8). When the service time follows the gamma $(p)$ distribution function

$$
\left[\mu^{p} / \Gamma(p)\right) \exp (-\mu x) x^{p-1} d x \quad(\mu>0 ; x \in(0, \infty)),
$$


then we get

$$
\Phi(\theta)=\mu^{p} /(\mu+\theta)^{p}
$$

so that we can decompose (3.8) in the form

$$
\varphi(\theta)=1-\delta+(1-\delta) \frac{\sum_{i=0}^{p-1} a_{i} \theta^{i}}{\sum_{i=0}^{p} b_{i} \theta^{i}},
$$

where $a_{i}, b_{i}, \ldots$ are constants. Hence we get

$$
\operatorname{prob}(W=0)=1-\delta
$$

and

$$
\varphi^{+}(\theta)=\sum_{i=1}^{k} c_{i} /\left(d_{i}+\theta\right),
$$

where $c_{i}, d_{i}$ are constants $(i=1, \ldots, p)$. Hence

$$
\operatorname{prob}(w>x)=\sum_{i=1}^{p} c_{i} \exp \left(-d_{i} x\right),
$$

where $c_{i}, d_{i}$ are complex-valued.

It may be interesting to investigate the nature of poles of $\varphi^{+}(\cdot)$ in (4.5). The following can be proved without difficulty. .

(i) For $p=2$, the poles of $\varphi^{+}(\cdot)$ in (4.5) are both real-valued and negative. Hence the waiting time distribution function is hyperexponential - both $d_{1}$ and $d_{2}$ in (4.5) are real.

(ii) For $p=3$, only one pole of $\varphi^{+}(\cdot)$ is real and there is a pair of complex-valued poles, so that

(4.6) $\quad H(x)=\operatorname{prob}(W>x)=c_{1} \exp \left(-d_{1} x\right)+2 c_{2} \exp (-m x) \cos q x$,

where the real valued pole is $-d_{1}$ and the pair of complex poles is $-m \pm j q(j=V-1)$. Thus $H(x)$ is a mixture of exponential distribution functions and a damped vibration.

(iii) For $p=4$, two poles of $\varphi^{+}(\cdot)$ are real and there is a pair 
of complex-valued poles: and so on.

EXAMPLE. Let $p=2, \alpha=0.4, \mu=0.9$, so that $\delta=0.889$. We get

$$
\begin{aligned}
\varphi^{+}(\theta) & =0.111(0.4 \theta+0.72) /\left(\theta^{2}+1.4 \theta+0.09\right) \\
& =0.405 /(\theta+1.3325)+0.0039 /(\theta+0.676),
\end{aligned}
$$

so that

$$
\operatorname{prob}(W=0)=0.111
$$

and

$$
d F(x)=0.405 \exp (-1.3325 x)+0.0039 \exp (-.0676 x) .
$$

From studies by Lilliefors [10] and the experimental studies of the present author, we know that an $M / G / 1$ system can be approximated by an $M / E_{p} / 1$ system. The choice of $p$ can be done in a heuristic way. Hence we have the following theorem.

THEOREM 4.1. For an $M / G / 1$ system we can in most cases get an $M / E_{p} / 1$ quasi-isomorph with respect to the waiting time distribution. In other words, we can choose $p$ such that the waiting time distribution function of the $M / E_{p} / 1$ system is almost equivalent to that of the $M / G / 1$ system - and it follows a generalized hyperexponential distribution.

NOTE. It can be shown without difficulty that such a quasi-isomorph of type $M / E_{p} / 1$ which approximates the waiting time distribution function will also approximate the other outputs, for example idle time distribution function, departure process, and so on. However, experiments have shown (not reported here) that when $p$ is large (say $6,7, \ldots$ ), the optimal quasi-isomorph with respect to the waiting time distribution function may not be optimal with respect to the idle time distribution function. For example, the optimal quasi-isomorph with respect to waiting time distribution functions may have a value of $p=5$, while the optimal quasi-isomorph with respect to the idle time distribution function may have a value $p=7$, and so on.

Theorem 4.1 is somewhat like a continuity theorem for $M / G / 1$ systems (for more elaborate works in continuity theorems, see Kennedy [8], Whitt [15]). 


\subsection{EXISTENCE OF AN $M / M / 1$ QUASI-ISOMORPH}

We state the following theorem regarding approximation of any general single-server system $G I / G / 1$ system by an $M / M / 1$ quasi-isomorph with respect to the waiting time distribution function.

THEOREM 4.2. A GI/G/1 system can be approximated by an $M / M / 1$ quasi-isomorph, with respect to the waiting time distribution function, In $H(x)$, where $H(x)=\operatorname{prob}(W>x)$, can be approximated by a linear form

$$
\text { In } H(x)=-a-b x \text {, }
$$

where $a(<1)$ and $b$ are positive numbers.

Proof. If (4.7) holds good, then the waiting time follows an exponential distribution, which is uniquely identified by $a$ and $b$ :

$$
H(x)=c \exp (-b x)
$$

where $c=\exp (-a)$. Thus we have to have the observed $H^{0}(x)$ in the form

$$
\ln H^{O}(x)=-a-b x+e,
$$

where $e$ is the error. It has to satisfy, for a good fit, the hypothesis that $e=0$; we may test the null hypothesis that $e$ follows a normal distribution with zero mean and unit standard deviation.

Theorem 4.2 Ieads to a useful graphical test. If we plot the results of an actual or experimented simulation, and find that $H(x)$ follows approximately a linear form on a logarithmic scale, then we can have an $M / M / 1$ quasi (or quasi-restricted) isomorph with respect to the waiting time distribution.

It can be easily proved that we can obtain an $M / M / 1$ restricted isomorph for a $G I / M / s$ system. From the results of Kendall [7], the interpretation in terms of isomorphs would be that, unlike the situation in a $G I / M / 1$ system, the isomorph with respect to the waiting time distribution function will not be the same as the isomorph with respect to the queue size distribution function because in a $G I / M / s$ system, $\operatorname{prob}(W=0)$ is not the same as $\operatorname{prob}(Q=0)$.

\section{Application in queues in series}

The real utility of the approach of approximation through isomorphic 
systems (in the $R$-sense or quasi- sense) lies in complex queueing systems like series queues, parallel queues, and so on. We can easily determine the waiting time distribution function in the second queue of a series of two queues $M / M / 1 \rightarrow \cdot / G / 1$, because the second queue behaves as a queue $M / G / 1$ where the inter-arrival time has the same exponential distribution function as the system of two queues in series (see Ghosal [3]). Approximations have been worked for an $M / E_{2} / 1 \rightarrow \cdot / M / 1$ system (Ghosal [2]). It is conjectured by the present author that the waiting time in the second queue in a two-queue series $M / E_{p} / 1 \rightarrow \cdot / E_{q} / 1$ system follows approximately a generalized hyperexponential distribution function of order $p+q$ (detailed discourses will be presented in subsequent publications and also in the monograph by the present author, Applied cybernetics: its relevance in operations research, to be published by Gordon and Breach, London and New York).

Interesting experiments have been done by the students of the present author (Affisco (unpublished) and Dan Shimshak, PhD thesis, Department of Management, Baruch College, New York - see also Shimshak [12]) on series queueing systems. It has been found from these experiments that in a large number of cases we can approximate the waiting time in a $G I / G / 1 \rightarrow \cdot / G / 1$ system (both for the waiting time in the second queue $w^{(2)}$ or the sum of waiting times in two queues $W^{(1)}+W^{(2)}$ ) by an $M / M / 1$ isomorph. In all such experiments our approach was to determine the error in such simplification.

In one experiment done by the author with a sample of 509 observations for estimating the distribution function of the waiting time distribution function in the third queue in an assembly process, the waiting time distribution function $H(x)$ was estimated by the hyperexponential distribution

$$
H(x)=0.25 \exp (-1.20 x)+0.46 \exp (-0.17 x), x>0 .
$$

The goodness of fit was tested by the Kolmogoroff-Smirnov test (see Massey [11]) against the observed $H^{0}(x)$ :

$$
D=\max _{x}\left|H^{0}(x)-H(x)\right|
$$

where the $\operatorname{prob}(W>x)$ was observed through a frequency distribution with 
time intervals of 0.4 minutes. The value of $D$ was 0.59 , which was less than the critical value at $P=.05$, which was $1.36 / n^{\frac{1}{2}}$. This suggested a reasonably good fit. The distribution function $H(x)$ could be approximated by an $M / E_{2} / I$ quasi-isomorph, in which $E($ inter-arrival time) equals 2.646 , and $E$ (service time) equals 1.26 minutes.

Another approach was to get upper and lower bounds of $M / M / 1$ or $M / H_{p} / 1$ or $M / E_{p} / 1$ isomorphs for network queues by two alternative methods - one used by Ghosal [5], which is an extension of Marshall's methods (see [15]) in queues in series, the other used by Shimshak and Sphicas (unpublished) by a set of inequalities in terms of coefficients of variation.

\section{Concluding remarks}

This paper gives some exploratory research done for solving approximately complex or analytically intractible queueing models with the help of equivalent or isomorphic systems. Isomorphisms have been used in a special sense - restricted and quasi-isomorphs have been obtained for a few cases. An isomorph which may give an equivalent waiting time distribution function may show shifts in the distribution function of queue size or idle time - in some cases shifts in the expectation and variance in these can be estimated. Seventy years' research on queueing systems indicates that analytical solutions are obtained in onlyalimited number of cases. Future research lies in innovating methods of approximation - the approach of deriving $R$-isomorphs or quasi-isomorphs appears to be a promising field of research not only in queueing systems but in statistical systems at large.

\section{References}

[1] P.D. Finch, "The output process of the queueing system M/G/I ", J. Roy. Statist. Soc. Ser. B 21 (1959), 375-380.

[2] A. Ghosal, "Queues in series", J. Roy. Statist. Soc. Ser. B 24 $(1962), 359-363$. 
[3] A. Ghosal, Some aspects of queueing and storage systems (Lecture Notes in Operations Research and Mathematical Systems, 23. Springer-Verlag, Berlin, Heidelberg, New York, 1970).

[4] A. Ghosal, "Cybernetic queueing systems I", Cahiers Centre Études Recherche Opér. 13 (1971), 183-202.

[5] A. Ghosal, "Cybernetic modelling of queueing and storage systems", SCIMA 4 (1975), 5-18.

[6] A. Ghosal, Isomorphic queues and related problems (Working Paper No. Gl/76. Graduate Center of Management, Baruch College, City University of New York, New York, 1976).

[7] David G. KendalI, "Stochastic processes occurring in the theory of queues and their analysis by the method of the imbedded Markov chain", Ann. Math. Statist. 4 (1953), 338-354.

[8] Douglas P. Kennedy, The continuity of the single server queue (Technical Report, 12. Department of Operations Research, Stanford University, Stanford, California, 1970).

[9] J. Klîr, M. Valach, Cybernetic modelzing (Illiffe, Iondon, 1965).

[10] Hubert W. Lilliefors, "Some confidence intervals for queues", operations Res. 14 (1966), 723-727.

[11] Frank J. Massey, Jr., "The Kolmogorov-Smirnov test for goodness of fit", J. Amer. Statist. Assoc. 46 (1951), 68-78.

[12] Daniel G. Shimshak, "Isomorphs of series queueing systems", SCIMA 5 $(1976), 57-73$.

[13] Walter M. Smith, "On the distribution of queueing times", Proc. Cambridge Phizos. Soc. 49 (1953), 449-461.

[14] Louis W. Stern, "A numerical study of queueing systems with normal inter-arrival process", SCIMA 4 (1975), 31-38.

[15] Ward Whitt, "Weak convergence theorems for queues in heavy traffic" (PhD thesis, Cornell University, New York, 1969. See also:

Technical Report, 2. Department of Operations Research, Stanford University, Stanford, California, 1968).

Council of Scientific and Industrial Research, Rafi Marg, New Delhi, India. 